\title{
Carrier-envelope phase stabilization of high-contrast femtosecond laser pulses with a relativistic intensity
}

\author{
Kyung-Han Hong ${ }^{\text {a) }}$ and Jongmin Lee \\ Femto Science Laboratory, Advanced Photonics Research Institute (APRI), Gwangju Insitute of Science and \\ Technology, 1 Oryong-dong, Buk-gu, Gwangju 500-712, Republic of Korea \\ Bixue Hou, John A. Nees, and Erik Power \\ FOCUS Center and Center for Ultrafast Optical Science, University of Michigan, 2200 Bonisteel \\ Boulevard, Ann Arbor, Michigan 48019 \\ Gerard A. Mourou \\ Laboratoire d'Optique Applique (LOA), Chemin de la Huniere, 91761 Palaiseau Cedex, France
}

(Received 13 February 2006; accepted 10 June 2006; published online 20 July 2006)

\begin{abstract}
We report on the generation of carrier-envelope phase (CEP)-stabilized pulses with a relativistic intensity and a high-contrast ratio. The CEP stabilization is achieved with a jitter of 0.95 rad from a $0.5 \mathrm{kHz}$ femtosecond laser pulses with a focal intensity of $2.6 \times 10^{18} \mathrm{~W} / \mathrm{cm}^{2}$ and a picosecond contrast of $2.5 \times 10^{-9}$. CEP noise analysis shows that the beam pointing at the pulse compressor is a dominant factor of the CEP fluctuation with our laser system. () 2006 American Institute of Physics. [DOI: 10.1063/1.2221877]
\end{abstract}

Recently, attosecond pulse generation and attosecond phenomena have attracted great attention among ultrafast laser researchers. Attosecond pulse generation using the highorder harmonic soft $\mathrm{x}$ rays driven by intense few-cycle femtosecond laser pulses ${ }^{1}$ has opened the way to attosecond metrology. ${ }^{2}$ One of the key technologies for the generation of reproducible single attosecond pulses using high-order harmonics is the stabilization of the carrier-envelope phase (CEP) of femtosecond laser pulses. The CEP locking techniques $^{3,4}$ applied to femotsecond mode-locked lasers, mainly developed for frequency metrology, have also made it possible to generate CEP-stabilized amplified pulses in chirped-pulse amplification (CPA) lasers. ${ }^{5}$ On the other hand, recent numerical studies show that attosecond pulses can be generated from relativistic interaction between femtosecond laser pulses and a dynamic plasma mirror ${ }^{6,7}$ or free electrons through nonlinear Thomson scattering. ${ }^{8}$ The dynamic-mirrorbased relativistic attosecond pulse generation can offer relatively high conversion efficiency even though the experimental conditions are more difficult to satisfy than those of conventional high-order harmonic generation scheme. According to these studies, CEP-stabilized few-cycle laser sources with a relativistic intensity are also required for the generation of an isolated attosecond pulse using plasma reflection schemes.

A "relativistic wavelength-cubed $\left(\lambda^{3}\right)$ " laser generating a relativistic intensity $\left(>2 \times 10^{18} \mathrm{~W} / \mathrm{cm}^{2}\right.$ in case of $800 \mathrm{~nm}$ wavelength) in a several-cycle (or few-cycle) pulse duration and a wavelength-limited spot size can be realized from a typical subterawatt kilohertz CPA laser with the help of a tight focusing technique using high-numerical-aperture optics and a wave-front correction method. ${ }^{9}$ The relativistic $\lambda^{3}$ regime is technically important because the generation of CEP-stabilized few-cycle laser pulses was already demonstrated with a conventional kilohertz CPA laser combined either by hollow-core fiber technique or by femtosecond fila-

\footnotetext{
${ }^{a)}$ Electronic mail: khhong@gist.ac.kr
}

mentation technique. ${ }^{10}$ Thus, a kilohertz $\lambda^{3} \mathrm{CPA}$ system is an available solution for the generation of CEP-stabilized relativistic laser pulses. Another important factor with a relativistic $\lambda^{3}$ laser is the intensity contrast ratio because the relativistically dynamic mirror is formed by overdense plasmas generated from a solid target. Recently, we have demonstrated a relativistic kilohertz $\lambda^{3}$ laser system with an amplified spontaneous emission (ASE) level of $<10^{-8}$ of the main pulse. $^{11}$

In this letter, we report on the CEP stabilization of the relativistic-intensity laser pulses with a high-contrast ratio. As illustrated in Fig. 1, our laser system contains three multipass amplifiers, a pulse cleaner made of a saturable absorber (SA) for high-contrast ratio, a grating stretcher, and a grating compressor. It is challenging to generate CEPstabilized pulses from this complicated laser system. The CEP stability of the relativistic laser pulse is measured using an $f$-to- $2 f$ spectral interferometer ${ }^{12}$ and the CEP jitter is quantitatively analyzed.

The main special feature of our high-contrast $0.5 \mathrm{kHz}$ relativistic laser is the addition of a nonchirped preamplifier and a 3-mm-thick SA (IR85 color filter, HOYA) to seed a

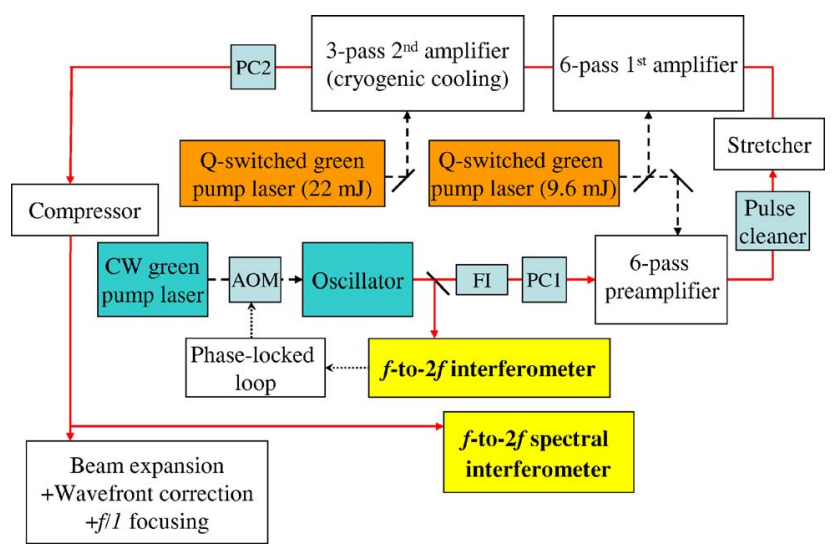

FIG. 1. Block diagram of high-contrast relativistic kilohertz laser. Acoustooptic modulator (AOM), Faraday isolator (FI), and Pockels cells (PCs). 


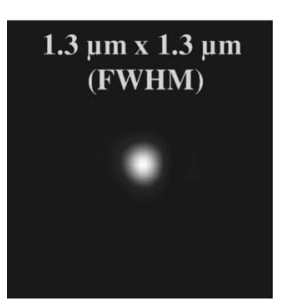

(a)

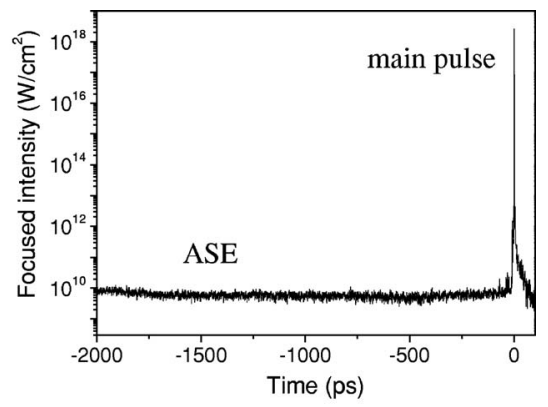

(b)
FIG. 2. Focal spot shape (a) and high-dynamic-range temporal structure (b).

clean high-energy (microjoule) pulses to the CPA chain. The laser pulse cleaned at the SA is stretched to $\sim 40 \mathrm{ps}$ in a grating stretcher and sequentially amplified at two multipass amplifiers up to $7.5 \mathrm{~mJ}$. Detailed laser parameters are described in Ref. 11. By focusing the compressed laser pulse with the duration of $29 \mathrm{fs}$ and the energy of $3.0 \mathrm{~mJ}$ down to the area of $1.3 \times 1.3 \mu \mathrm{m}^{2}$ at full width at half maximum (FWHM) using an adaptive optic system, we have achieved a relativistic laser intensity of $2.6 \times 10^{18} \mathrm{~W} / \mathrm{cm}^{2}$. The ASE level in the picosecond range measured using a highdynamic-range third-order cross correlator is as low as 2.5 $\times 10^{-9}$. The beam shape and the high-dynamic-range temporal profile at the focal spot are shown in Figs. 2(a) and 2(b), respectively. Figure 2(b) is modified from the third-order cross-correlation measurement.

For the CEP stabilization of the high-contrast relativistic laser pulses, we have installed a commercial phase stabilization system (XPS800, Menlosystem $\mathrm{GmbH}$ ) for our laser oscillator. This system utilizes an $f$-to- $2 f$ interferometer for the measurement of the shot-to-shot CEP change $(\Delta \phi)$ and a phase-locked loop for the stabilization. Actually, we have locked $\Delta \phi$ to $\pi / 2$ by locking the $f$-to- $2 f$ beat frequency $\left(f_{\text {ceo }}\right)$ to $1 / 4$ the repetition rate $\left(f_{\text {rep }}\right)$, where $\Delta \phi$ is given by $2 \pi\left(f_{\text {ceo }} / f_{\text {rep }}\right)$. Figure 3 (a) shows the rf spectrum of the $f$-to$2 f$ beat signal locked to $18 \mathrm{MHz}$, which is $1 / 4$ of the laser repetition rate, $72 \mathrm{MHz}$. The power of our pump laser (Millennia, Spectra Physics Inc.) is slightly modulated using an acousto-optic modulator for a feedback control, as shown in Fig. 1. The rms phase noise for $\sim 1 \mathrm{~s}$ is estimated as $\sim 0.3 \mathrm{rad}$ from Fig. 3(b), which is an accumulated oscilloscope trace of the beat signal, synchronized to the modelocked pulse train. Using a synchronized frequency divider and a Pockels cell with two polarizers, we have selectively

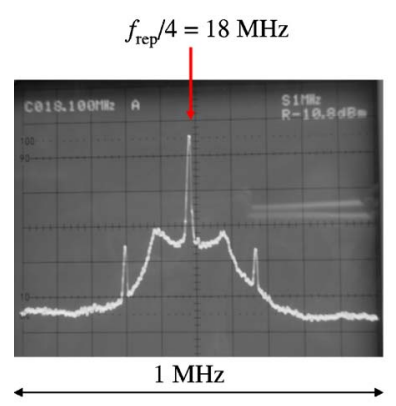

(a)

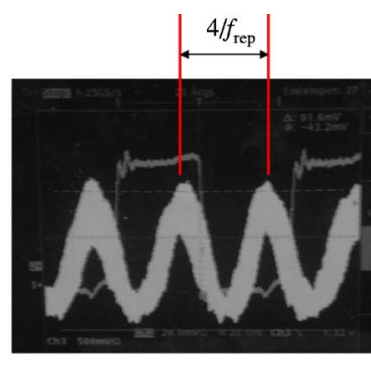

(b)
FIG. 3. rf spectrum of $f$-to- $2 f$ beat signal (a) and phase noise (b) accumulated over $\sim 1 \mathrm{~s}$. The square signal with $f=f_{\text {rep }} / 8$ in (b) is synchronized to mode-locked pulse train.
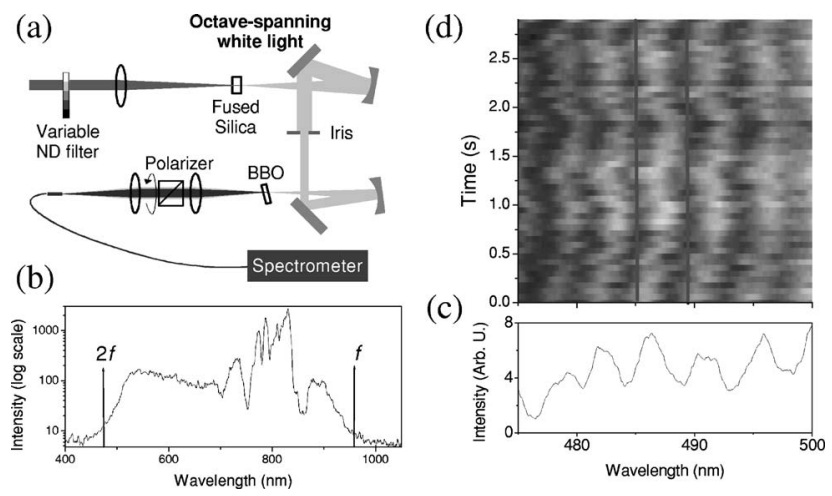

FIG. 4. Optical setup of $f$-to- $2 f$ spectral interferometer (a), generated continuum spectrum (b), measured spectral interferogram (c), and time evolution of the interferograms (d).

amplified the laser pulses with the same CEP at $0.5 \mathrm{kHz}$.

The CEP-stabilized laser pulses are amplified up to $\sim 1 \mu \mathrm{J}$ and then focused on the SA for pulse cleaning. Saturable absorption is an intensity-dependent nonlinear process, but it induces only amplitude modulation without phase modulation unlike optical Kerr effect. Hence, the CEP of the amplified pulses can be maintained on a SA. Moreover, the amplified laser pulse saturates the SA and, thus, the shot-toshot intensity fluctuation ( $3 \% \mathrm{rms}$ ) is not magnified by the SA. Nevertheless, the amplitude-to-phase conversion of the CEP noise can still occur by means of Kerr nonlinearity. B-integral value of the laser pulse at the SA is estimated to be $0.1 \mathrm{rad}$ or less, resulting in a CEP jitter of $\sim 10^{-3} \mathrm{rad}^{13}$ Therefore, we can safely use the SA for pulse cleaning with the CEP being maintained.

To measure the shot-to-shot CEP stability of the amplified and compressed pulse, we have also set up an $f$-to- $2 f$ spectral interferometer, ${ }^{12}$ as shown in Fig. 4(a). The $\sim 1 \mu \mathrm{J}$ pulse leaked from the main pulse generates a one-octavespanning continuum on a 3-mm-thick fused silica block with $\sim f / 20$ focusing, as shown in Fig. 4(b). Beam size and energy are finely controlled to obtain a good spatial quality with a single filament. The generated continuum is collimated using a concave mirror, spatially filtered by an aperture, and then focused on a 0.3-mm-thick type I beta barium borate crystal. A second harmonic signal of long-wavelength part, i.e., $960 \mathrm{~nm}$, of the continuum, is interfered with the short-wavelength part, i.e., $480 \mathrm{~nm}$. The polarizer makes it possible to observe a spectral interference between two orthogonally polarized signals. The measured $f$-to- $2 f$ spectral fringe is single shot based and shown in Fig. 4(c). We can measure the shot-to-shot CEP noise by monitoring the time evolution of this fringe.

We have observed the stabilized interferograms for $\sim 3 \mathrm{~s}$, as shown in Fig. 4(d), whereas the interferograms without the CEP locking of the oscillator laser pulses reveal a random feature. The CEP jitter of the stabilized pulses, calculated from Fig. 4(d), is 0.95 rad in rms. Even though the phase jitter is relatively large compared to those of more simple laser systems in which the CEP locking has been demonstrated, ${ }^{1}$ our result shows that CEP stabilization process can be extended to a more complicate laser system like our laser. Moreover, we have demonstrated the CEP stabilization of relativistic laser pulses with a high-contrast ratio.

In the measurement of the CEP fluctuation, the amplitude-to-phase conversion at the $f$-to- $2 f$ spectral inter- 


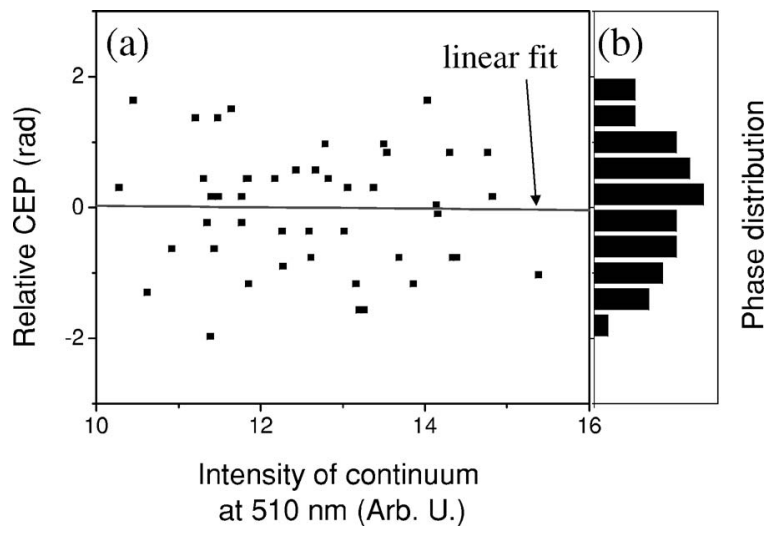

FIG. 5. Intensity dependence of relative CEP to estimate the amplitude-tophase conversion noise at the interferometer (a) and the corresponding CEP distribution (b)

ferometer should be low enough. To analyze this effect, we have plotted the statistics of the relative CEP with regard to the intensity of continuum, as shown in Fig. 5(a). The corresponding phase distribution is shown as Fig. 5(b). The wavelength of $510 \mathrm{~nm}$, where there is no spectral interference, is monitored as a reference position for the intensity axis because the spectrum fluctuation of the continuum directly reflects the intensity fluctuation of input laser pulses. The intensity at $510 \mathrm{~nm}$ is observed to be proportional to the input laser intensity because this wavelength is far from the central part of amplified laser spectrum (at around $800 \mathrm{~nm}$ ) where an intensity-dependent modulation structure appears, as seen in Fig. 4(b). The correlation curve with a linear fit in Fig. 5(a) reveals that the CEP and the laser intensity are irrelevant to each other. Thus, the amplitude-to-phase conversion at the $f$-to- $2 f$ spectral interferometer can be neglected.

Low phase jitter and good long-term stability are important for the experimental applications. Baltuska et al. $.^{5} \mathrm{ob}-$ tained the phase jitter less than $0.1 \mathrm{rad}$ for $\gg 1 \mathrm{~min}$ in a single-amplifier system without any grating stretcher or compressor with the help of an additional slow feedback method. However, grating-based CPA systems have more problems in the CEP stabilization because the beam pointing can sensitively change the shot-to-shot and long-term CEP. Even though the roundtrip geometry in a grating stretcher or in a grating compressor can compensate the CEP fluctuation to some extent, ${ }^{13}$ the beam pointing stability is still the biggest issue. In our case, the beam pointing fluctuation at the stretcher is $<1 \mu \mathrm{rad}$ in rms while that measured at the compressor is as large as $\sim 20 \mu \mathrm{rad}$. The CEP sensitivity with regard to the angular deviation in the compressor is calculated as $1.2 \times 10^{6} \mathrm{rad} / \mathrm{rad}$ for single pass, implying that the CEP jitter is expected to be $\sim 24 \mathrm{rad}$. However, the experimental CEP sensitivity from measured CEP jitter (0.95 rad) is only $4.8 \times 10^{4} \mathrm{rad} / \mathrm{rad}$ because of the geometric CEP compensation effect at the compressor. This result is quantitatively consistent to the previous studies ${ }^{13,14}$ and indirectly shows that our CEP jitter is limited by the beam pointing at the compressor.

Several other factors should be considered for the analysis of CEP stability. Currently, the CEP jitter of the oscillator pulses is not the main factor for the CEP jitter of the compressed pulses, but it can give a fundamental limitation even after enhancing the beam pointing stability. Actually, we have obtained at least five times lower CEP jitter of the oscillator pulses by replacing the pump laser by a single-mode green laser. Second, the CEP change due to the nonlinear phase fluctuation in the amplifier media can be randomly generated, but this is less than $10^{-2}$ rad for the B-integral less than $1 \mathrm{rad}^{13}$ Third, a thermal drift should be considered when a good long-term stability for $\gg 1 \mathrm{~min}$ is necessary, whereas it can be neglected in our measurement $(\ll 1 \mathrm{~min})$. Therefore, the beam pointing problem is still the most dominant factor of CEP fluctuation of our CPA system. The beam pointing stability can be enhanced by using a regenerative amplifier as the first multipass amplifier and minimizing the air flow inside the laser system. Downchirped-pulse amplification schemes ${ }^{15,16}$ can also relax this problem.

In conclusion, we have demonstrated the generation of CEP-stabilized laser pulses with a relativistic intensity and a high-contrast ratio. Better pointing stability is required for low phase jitter and good long-term stability. This laser will become a more promising source for the relativistic $\lambda^{3}$ experiments if the slow feedback method and a high-energy pulse compression technique preserving the CEP are accompanied.

This work was supported by the NSF (FOCUS Program, Grant No. 0114336) and the ARO (Grant Nos. DAAD19-031-0287 and DAAD19-03-1-0316). One of the authors (K.H.H.) would wish to thank Korea Research Foundation (KRF) for its support through the postdoctoral Fellowship Program.

${ }^{1}$ A. Baltuska, Th. Udem, M. Uiberacker, M. Hentschel, E. Goulielmakis, Ch. Gohle, R. Holzwarth, V. S. Yakovlev, A. Scrinzi, T. W. Hansch, and F. Krausz, Nature (London) 421, 611 (2003).

${ }^{2}$ M. Drescher, M. Hentschel, R. Kienberger, M. Uiberacker, V. Yakovlev, A. Scrinzi, T. Westerwalbesloh, U. Kleineberg, U. Heinzmann, and F. Krausz, Nature (London) 419, 803 (2002).

${ }^{3}$ D. J. Jones, S. A. Diddams, J. K. Ranka, A. Stentz, R. S. Windeler, J. L. Hall, and S. T. Cundiff, Science 288, 635 (2000).

${ }^{4}$ Y. S. Lee, J. H. Sung, C. H. Nam, T. J. Yu, and K.-H. Hong, Opt. Express 13, 2969 (2005).

${ }^{5}$ A. Baltuska, M. Uiberacker, E. Goulielmakis, R. Kienberger, V. S. Yakovlev, Th. Udem, T. W. Hansch, and F. Krausz, IEEE J. Sel. Top. Quantum Electron. 9, 972 (2003).

${ }^{6}$ S. V. Bulanov, T. Esirkepov, and T. Tajima, Phys. Rev. Lett. 91, 085001 (2003).

${ }^{7}$ N. Naumova, J. A. Nees, I. V. Sokolov, B. Hou, and G. A. Mourou, Phys. Rev. Lett. 92, 063902 (2004).

${ }^{8}$ K. Lee, Y. Cha, M. Shin, B. Kim, and D. Kim, Opt. Express 11, 309 (2003).

${ }^{9}$ O. Albert, H. Wang, D. Liu, Z. Chang, and G. Mourou, Opt. Lett. 25, 1125 (2000).

${ }^{10}$ C. P. Hauri, W. Kornelis, F. W. Helbing, A. Heinrich, A. Couairon, A. Mysyrowicz, J. Beigert, and U. Keller, Appl. Phys. B: Lasers Opt. 79, 673 (2004).

${ }^{11}$ K.-H. Hong, B. Hou, J. A. Nees, E. Power, and G. A. Mourou, Appl. Phys. B: Lasers Opt. 81, 447 (2005).

${ }^{12}$ M. Kakehata, H. Takada, Y. Kobayashi, K. Torizuka, Y. Fujihira, T. Homman, and H. Takahashi, Opt. Lett. 26, 1436 (2001).

${ }^{13}$ M. Kakehata, Y. Fujihira, H. Takada, Y. Kobayashi, K. Torizuka, T. Homman, and H. Takahashi, Appl. Phys. B: Lasers Opt. 74, S43 (2002).

${ }^{14}$ I. Thomann, E. Gagnon, R. J. Jones, A. S. Sandhu, A. Lytle, R. Anderson, J. Ye, M. Murnane, and H. Kapteyn, Opt. Express 12, 3493 (2004).

${ }^{15}$ D. M. Gaudiosi, A. L. Lytle, P. Kohl, M. M. Murnane, H. C. Kapteyn, and S. Backus, Opt. Lett. 29, 2665 (2004).

${ }^{16}$ K.-H. Hong, S. Kostritsa, T. J. Yu, J. H. Sung, I. W. Choi, Y.-C. Noh, D.-K. Ko, and J. Lee, Opt. Express 14, 970 (2006). 